\title{
Research of Parking Management System Based on Centralized Control Yaojie Chen ${ }^{1,}$, Min $\mathrm{Su}^{2, \mathrm{~b}^{*}}$ and Ming $\mathrm{Qi}^{3, \mathrm{c}}$ \\ ${ }^{1}$ College of Computer Science and Technology, Hubei Province Key Laboratory of Intelligent Information Processing and Real-time Industrial System, Wuhan University of Science and Technology, Wuhan 430065, Hubei, China; \\ ${ }^{2}$ College of Computer Science and Technology, Wuhan University of Science and Technology, Wuhan 430065, Hubei, China

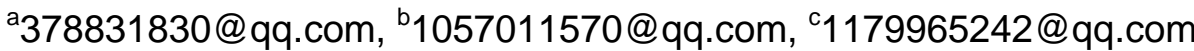 \\ ${ }^{*}$ The corresponding author
}

Keywords: Centralized control; B/S; C/S; Windows service

\begin{abstract}
In order to avoid the shortage in the parking lot management system in market at present with $\mathrm{C} / \mathrm{S}$ mode, such as upgrade difficult and high cost and the system rely on computers in workstations, this paper proposed an improved system with B/S mode based on centralized control. In the design, centralized control will be the network topology and the system uses a specialized windows service to receive and process data generated by the system. With a lot of tests and practicalities, it has been proved that this new parking management system is more stable while the cost is decreased.
\end{abstract}

\section{Introduction}

At present, most Parking Management System uses the Client and Server Structure. With this method, you can make full use of the processing capacity in the workstation computers. And then save the data in the server after been handled in the client. But this method also has some disadvantages like upgrade difficult and high cost and the system rely on computers in the workstations. When the computers in workstation broke down, the whole system cannot work in correct.

To solve the shortage of the parking management system with client and server structure, this paper proposed a improve system based on browser and server structure and Centralized Control. Under the centralized control mode, all the workstations have been managed by central control terminal. System workstation is used to monitor entrance and display parking fees, and problems appear in workstations will not affect the function of the entire system. In this paper, the system used the MVC (Model View Controller) design patterns, MVC model can help manage this complex system, but also simplifies the grouping development, shorten the development cycle. And, we use special windows service to receive and process data, making the data in system interact more efficient and stable.

\section{Hardware Design}

Now, most product of parking management system in the market uses the topology like Fig. 1 as shown below. 


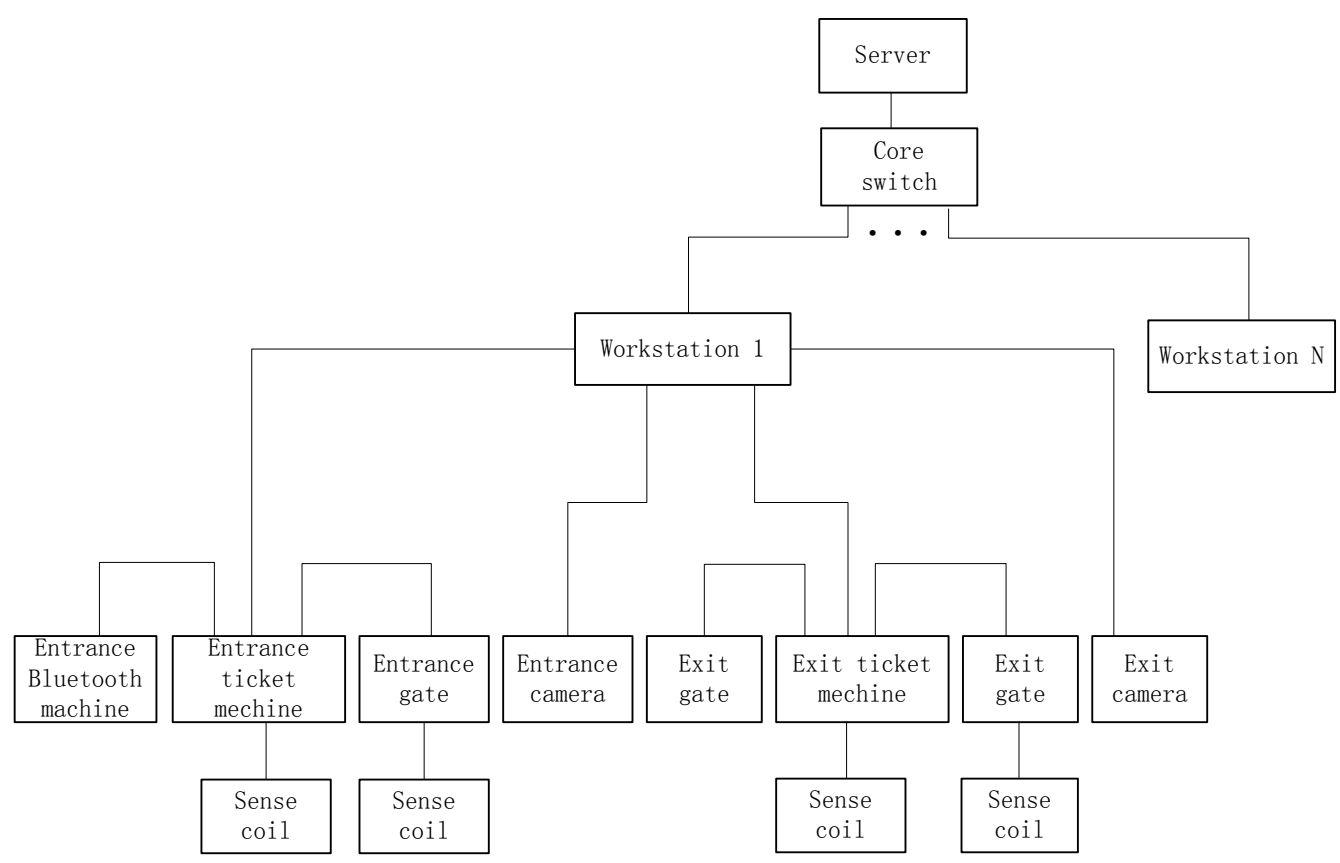

Figure 1. Network topology of system in the market.

In this control mode, the workstation is directly control on the entrance and exit, it plays a vital role, once there is a problem such as a workstation computer no power or other error, and then the gateway managed by this workstation will not work.

The network topology of system has been improved like Fig. 2 as shown below.

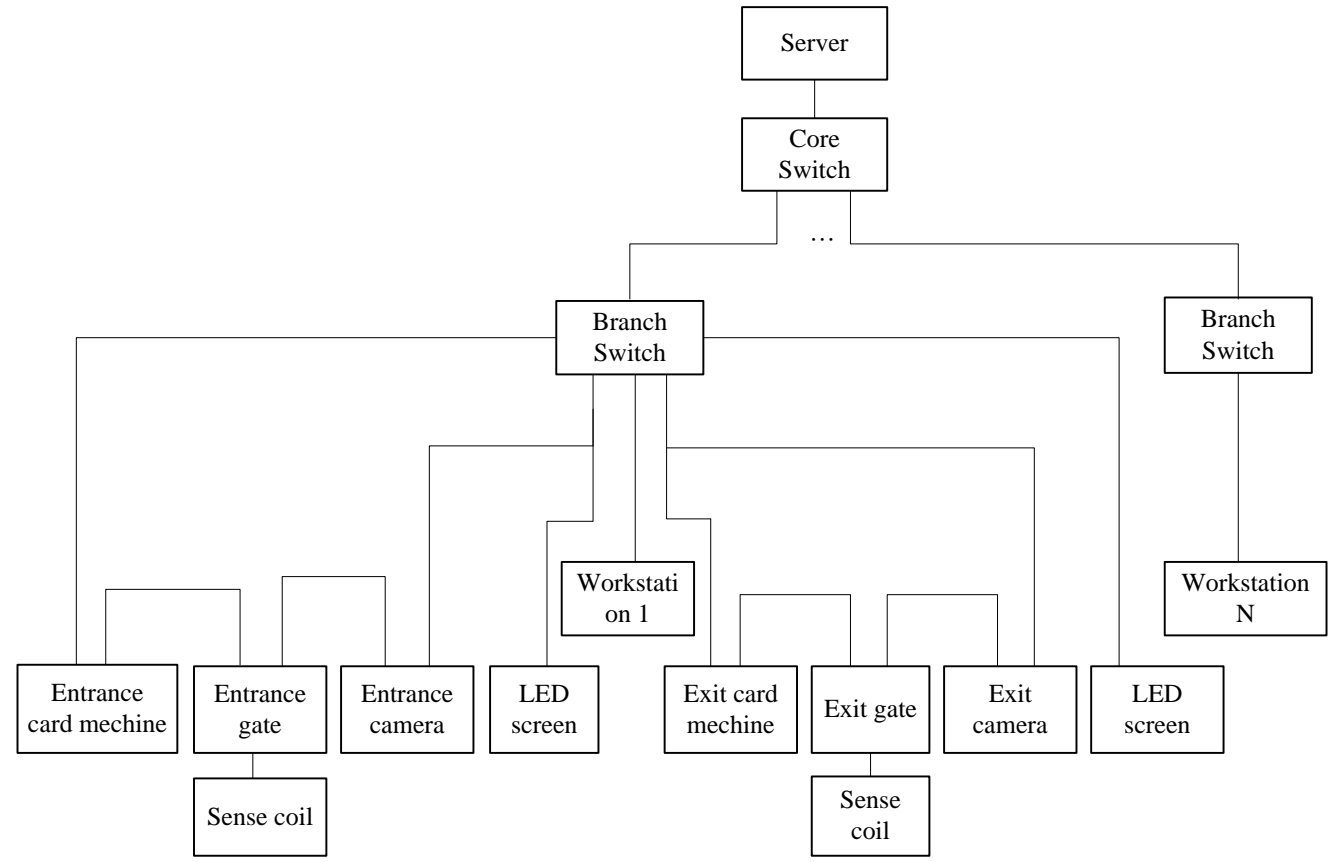

Figure 2. Network topology of system in the paper.

The difference of the system between market and this paper is that the other devices and workstations are in a same level. In traditional system, the workstation is on top of other devices in network topology. When in the face of unexpected situations, the improved one have more advantages as follows. Any device or workstation problems in the entire topology will not affect the system. For example, when camera cannot distinguish license plate, we can get a card in issuing machine. When workstation error, supplementary device of this workstation can send data to other workstations to handle, then it can promptly adjust the exit and entrance channels to make sure the system normal operation. 


\section{Software Design}

System Function. In this paper, the parking management system use automatic license plate recognition technology and automatic control technology to complete complex work, such as parking fees management and vehicle control. Vehicle traffic crossing the implementation of access control, surveillance, parking meters and other integrated management. The system implement centralized monitoring and charge in workstation, and license plate recognition technology will be used in exports and entrance. And then the parking system will be established to a convenience and safe control system. The system in this paper has a lot of features, such as, vehicle management, vehicle authentication and so on. The system's work flow chart like Fig. 3 as shown below.

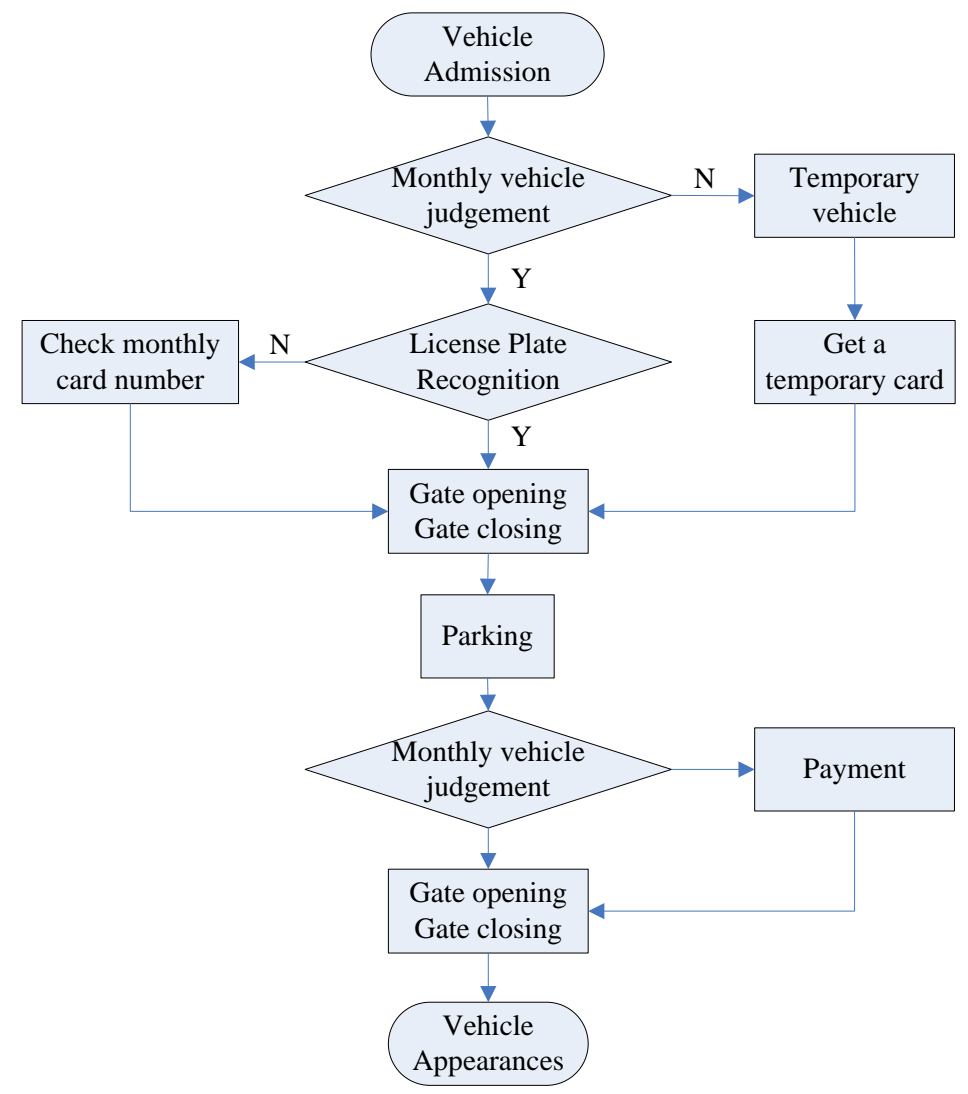

Figure 3. System's Work flow chart

When vehicles into the parking lot, first, if this car is the regular membership, license plate recognition system will record the plate and let the car into parking lot, and if this car is the temporary member, it can get a temporary card to into the parking lot. When vehicles exit the parking lot, regular membership will be free and others should be pay.

Data Transmission. The system in market process data in workstations and then upload the data to servers. Compared with this data processing method, the system in this paper use workstations and grouping equipment to collect data, then send data to a central control computer. A specialized windows service was been programmed to deal with data, and part of the data has been send back to workstations or backup on servers. Windows service has a good stability, as long as the computer with this service does not collapse, the service will work stably. This data processing greatly improves the stability of the management system, reducing the system response time, and have a good user experience. Windows service communicated with other devices like cameras and card machines to collect data and save data. Windows service can auto start when operation system start, and it don't have user interface so less resources it needs. The data transmission in this paper and in market like Fig. 4 as shown below. 


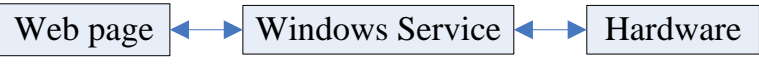

(a)Data transmission of system in this paper

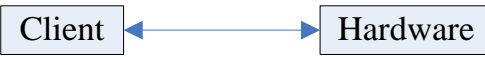

(b)Data transmission of system in market

Figure 4. System's data transmission

As shown in Fig. 4, this paper communicated with hardware by service, not client can reduce workstation's pressure and system's cost, and also avoids problems like data change failed caused by client been closed or breakdown.

System Structure. In this paper, Browser and Server mode will be the system structure. This method can avoid inadequate in client and server mode.

First, the system in this paper can be easy when maintain and update. Nowadays, software also update frequently, products with browser and server mode can be more convenient. When system needs update, products with client and server mode will waste a lot of time to reinstall all clients. And this system have several management mode, if they need modified, all clients need modified, if don't do that, data consistency will be destroyed. The system in this paper is very easy when software update and can also avoid shortage of client and server mode. Developers can update and maintain this system at server and then all workstations access the same code. Under this method, system consistency is guaranteed, so system cans normal operation.

Second, system's stability has been greatly improved. Under client and server mode, client has been set up on computers in workstations. Users of system mostly are not professional; their misuse may cause client closed or system breakdown. The system in this paper is browser and server mode, and all business logic has been put on server, and then backup server can reduce the possibility of system.

Third, cost reduction. This system can be installed on the Linux server and its high security. At the same time, regardless of the server selected which operating system can make use of windows operating system users are not affected.

\section{System Test}

By summarizing the same parking management system products, we can get several common indicators used to measure system performance: first, quantity of vehicles can system managed. Second, system's response speed. Third, length of historical data record. Fourth, no fault operation hours. To test the system functions, first, do the function test for every module in system. Second, do the integration test for some modules which rely on each other. No problems found during testing, the system performed well. At last, we set up the system at some communities to do the beta test. This community has three hundred parking spaces, two entrances and two exits. This paper choose system's response speed in several common indicators for standard and then use a tool named iYun to test the response speed of the system and other websites like Baidu and Google and so on. Then got the following set of data in table 1 .

Table 1 Response speed of the system and other websites

\begin{tabular}{ccccc}
\hline Website & Total time $(\mathrm{ms})$ & $\begin{array}{c}\text { Resolution } \\
\text { time }(\mathrm{ms})\end{array}$ & $\begin{array}{c}\text { Connection } \\
\text { time }(\mathrm{ms})\end{array}$ & Download time $(\mathrm{ms})$ \\
\hline Baidu & 32.77 & 27.68 & 0.46 & 4.63 \\
Tencent & 49.04 & 28.25 & 4.06 & 16.73 \\
The & 39.36 & 31.78 & 2.17 & 5.41 \\
System & 178.03 & 28.44 & 8.87 & 140.72 \\
360 & & & & \\
\hline
\end{tabular}


As can be seen from Table 1, the system in this paper compared to other website, the response time is within the normal range, this means that this improvement is feasible.

\section{Conclusion}

This paper proposed and implemented a parking management system based on central-control. When system process data, devices of workstations collect data and then transmission them to server and returned to workstations after been processed by server. The whole system architecture using client and server mode, in the specific coding, system using MVC design pattern. Compared with the currently available parking system uses C / S model, system in this paper improving the stability, development efficiency and practicality of the system, reducing the system response time and reduces the cost of system maintenance and update, expected to reach the beginning of the system design Effect.

\section{Acknowledgements}

Modeling and Simulation of Ship Waves (2016znss09B);

\section{References}

[1] Chen Li-jia.: Decision on multi target ship intelligent collision avoidance at sea, School of Navigation, Wuhan University of Technology, Wuhan, Hubei, 2011.

[2] Lian Xiao-feng, Liu Zai-wen, Zuo Min.: Research on dynamic artificial potential field path planning for dynamic robot, Computer simulation, 2011, 28(1), pp.27-31.

[3] Liao Yu-lei, Pang Yong-jie, Wan Lei.: Combined speed and yaw control of under actuated unmanned surface vehicles//Proceedings of International Asia Conference on Informatics in Control, Automation and Robot-ics, 2010, pp.157-161.

[4] Caeeia M, Bibull M, Bono R.: Basie navigation guidance and control of an unmanned surface vehicle, Auto Robot, 2008, 25, pp. 349-365.

[5] Fiorinip, Shiller Z.: Motion planning in dynamic environments using velocity obstacles, The International Journal of Robotics Research, 1998, 17(7), pp. 760-772.

[6] Largel F, Sekhavat S, Shiller Z.: Towards real-time global motion planning in a dynamic environment using the NLVO Concept[C]//Proceedings of the 2002 IEEE/RSJ International Conference on Intelligent Robots\& Systems Lausanne, Switzerland, 2002.

[7] Liu He-xiang, Bian Xin-qin, Qin Zheng.: Autonomous underwater robot collision avoidance algorithm and its simulation, Computer simulation, 2007, 24(12), pp. 141-144.

[8] Huang Yong-long, Zhong Xun-xian.: The collision avoidance algorithm to improve the speed of the robot based on multi barrier method, Computer engineering and Application, 2012, 48(32), pp. 47-51.

[9] Lewandowski, Edward M.: The dynamics of marine craft. World Scientific Pub Co Inc, 2004.

[10] Yasuo Yoshimura.: Coasting Manoeuvr- ability of single CPP equipped ship and the application of a new CPP controller, Proc. of MARSIM'93, pp. 651-660, 1993.

[11]Liu Yu-hong.: Multi objective optimization model of calculating the rate of ships to avoid, Journal of Shanghai Maritime College, 2001, 12(22), pp. 45-48. 